\title{
Editorial: Structured abstracts: A key to enhanced information transfer
}

A primary goal of Physical Review $C$ is to disseminate nuclear physics knowledge to research scientists working in the field. As a component of that effort, each published paper should carry a thoughtful, well organized abstract that conveys the main elements of the paper including the pertinent results. In many cases, however, published papers carry abstracts with little or no useful content. The journal does not have the resources necessary to make amends and create a proper abstract.

In an effort to improve the information conveyed to readers, who are attracted sufficiently to a paper by its title and read the abstract that follows, we are requesting that all authors prepare a "structured abstract" for each paper submitted. Examples of structured abstracts can be found in other fields, in particular in medical papers. They can even be found in papers already published in Physical Review $C$. Now that REVTeX 4.1 fully supports structured abstracts (see introduction to REVTeX 4 at https://authors.aps.org/revtex4/), it is time to introduce them formally to the Physical Review C community on an interim voluntary basis. At a point in time in the future, structured abstracts will be required of all papers published in the journal. At the moment, authors should begin to employ them as a means to enhance the information transfer to their readers and to attract readers who would not otherwise read the paper.

A structured abstract contains sections summarizing the paper's Background, Purpose, Methods, Results, and Conclusions. One can examine the REVTeX 4.1 guides for details of implementation (see documentation at https://authors.aps.org/revtex4/). However, an example from a specific paper is perhaps more enlightening: N. Anantaraman et al., "Electron capture strength for. .," Phys. Rev. C 78, 065803 (2008), available at http://link.aps.org/abstract/PRC/v78/e065803/.

Structured abstract:

Background: The strength of electron capture for medium mass nuclei has a significant effect on the evolution of supernovae. There is insufficient knowledge of these strengths and very little data for important radioactive nuclei. Purpose: Determine whether it is feasible to obtain EC strength from studies of $T_{o}+1$ excitations in $(p, n)$ reactions, and whether this might yield information for radioactive nuclei. Methods: Cross sections for the ${ }^{58,60,62,64} \mathrm{Ni}(p, n){ }^{58,60,62,64} \mathrm{Cu}$ reactions were measured over the angular range of $0.3^{\circ}$ to $11.6^{\circ}$ at $134.3 \mathrm{MeV}$ using the IUCF neutron time-of-flight facility. Results: The $T_{o}+1$ excitations in ${ }^{60,62} \mathrm{Ni}$ were identified by comparison with inelastic proton scattering spectra, their $B(\mathrm{GT})$ were extracted, and the corresponding electron capture rates in supernovae were calculated. Data from the TRIUMF $(n, p)$ experiments at $198 \mathrm{MeV}$ were reanalyzed; the electron capture rates for the reanalyzed data are in moderately good agreement with the higher resolution $(p, n)$ results, but differ in detail. The possibility of future measurements with radioactive nuclei was considered. Conclusions: It may be possible to obtain low-lying electron capture strength for radioactive nuclei by studying $(p, n)$ reactions in inverse kinematics.

Normal abstract:

Cross sections for the ${ }^{58,60,62,64} \mathrm{Ni}(p, n)^{58,60,62,64} \mathrm{Cu}$ reactions were measured at 134.3 MeV. Electron capture (EC) strength was obtained for ${ }^{60,62} \mathrm{Ni}$ from the cross section for excitation of $T_{o}+1$ states in the product nuclei. This approach yields better resolution than the $(n, p)$ reaction but is subject to larger backgrounds. It should also be applicable to studies of radioactive nuclei; their EC strength is important for studies of supernova evolution.

We note that anecdotal studies have shown that readers are much more likely to read the paper after reading the structured abstract than after reading the normal abstract. Therefore, Physical Review $C$ will initiate use of structured abstracts in the journal by encouraging authors to prepare a structured abstract for all submitted papers. Structured abstracts will not become a required element of a paper published in the journal until a future date.

Benjamin F. Gibson

Editor

Published 29 September 2011

DOI: 10.1103/PhysRevC.84.030001

PACS number(s): 01.30.Ww, 01.20.+x 